\title{
Potential diagnostic markers for postpartum depression point out to altered immune signaling
}

\author{
Molecular Psychiatry (2010) 15, 1; \\ doi:10.1038/mp.2009.142
}

The field of psychiatry suffers from the lack of biological markers that can define early cases, offer prognostic information, and guide choice of treatment as well as treatment response. The fully established phenotypes in psychiatry are among the few in medicine that can sometimes be identified by a quick glance across the room: a diagnosis of chronic paranoid schizophrenia can be more rapidly established than that of most cancers. Where we fail is in the identification of cases early on, when treatment would be not just palliative, but could have a preventive component, if not completely avoiding the disease phenotype, at least rescuing it early enough to prevent long-term functional sequelae.

The first in-patient I treated in my psychiatry residency 25 years ago was a woman who dramatically confessed killing her baby. The child had been diagnosed dead by natural causes. In spite of considerable effort, it became impossible to ascertain whether the patient had indeed committed infanticide, or if the idea of the alleged infanticide was one of several psychotic delusions that were part of her severe postpartum depression that went undiagnosed for weeks. Even after successful treatment with electroconvulsive therapy, it was very hard for all involved - the patient, her family and the treatment teamto decide how to understand the baby's death: sudden infant death syndrome or infanticide? The matter was never resolved in anyone's mind. What a difference early treatment for postpartum depression could make!

In this issue of Molecular Psychiatry, Segman et al. ${ }^{1}$ present data showing that in a small number of very wellcharacterized cases a pattern of gene expression from peripheral blood mononuclear cells could differentiate at the time of birth women who developed postpartum depression from those who did not in $84 \%$ of cases. The gene expression markers they identified were along interesting biological pathways. Those developing persisting postpartum depression exhibit a relative downregulation of transcription after delivery, with differential immune activation, decreased transcriptional engagement in cell proliferation, and DNA replication and repair processes. These findings are of diagnostic relevance, but they also point out to new clues in the biology of postpartum depression.

Having just returned from the 10th Psychoimmunology Expert Meeting, organized by Karl Bechter and colleagues from the University of Ulm, that took place at Reisensburg Castle in Germany on 12-14 November 2009, I am particularly attuned to the immune components that are part of the signature that Segman identified for postpartum depression. Segman et al. showed differential expression of several interferon-induced proteins. We recently reported increased expression of the $10-\mathrm{kDa}$ interferongamma-induced protein CXCL10 in depressed patients; CXCL10 levels decreased back to normal after antidepressant treatment. ${ }^{2}$ On the basis of our genetic findings of association with $\mathrm{T}$ bet $(T B X 21)$ as well as the functional finding of increased CXCL10 activity, we proposed increased Th1 immune activity in depression. Of interest here, most of Segman's findings were of decreased gene expression in postpartum depression, while we reported increased levels of CXCL10. Such differences could be explained in many ways: We looked at different subtypes of depression (postpartum versus adult depression), in different populations (Israelis versus Mexican-Americans) and different outcome measures (gene expression versus genotype and protein levels). One could focus on the differences in findings, but what I see as remarkable is that in two vastly different populations, adult MexicanAmericans in Los Angeles and Israeli women with postpartum depression, after large screenings using different types of outcomes measures, findings converge at the level of interferon-induced proteins.

Since the 1980s we have been searching for alterations in central immune signaling in depression. The demonstration that cytokine genes are expressed in the brain ${ }^{3}$ and a body of data that accumulated ever since supporting the hypothesis that signaling mediated by immune molecules can underlie the biology of some subtypes of major depressive disorder. ${ }^{4}$ In that context, it is noteworthy to see that immune mediators are part of a molecular signature for postpartum depression that has potential as a basis for early diagnosis, and potentially leading to life-saving treatments for this devastating disorder. Such early interventions will prevent others from being uncertain for a quarter of a century as to whether the death of a child cared by a mother with postpartum depression was homicide or sudden infant death syndrome.

\section{J Licinio John Curtin School of Medical Research, Australian National University, Canberra, Australia E-mail: julio.licinio@anu.edu.au}

\footnotetext{
References

1 Segman RH et al. Mol Psychiatry 2010; 15: 93-100 (this issue).

2 Wong ML et al. Mol Psychiatry 2008; 13: 800-812.

3 Licinio J et al. Endocrinology 1991; 129: 562-564.

4 Licinio J, Wong ML. Mol Psychiatry 1999; 4: 317-327.
} 